\title{
Inelastic post-buckling GBT analysis of tubular thin-walled metal members
}

\author{
M. Abambres \\ Civil Engineering Department, ICIST, Instituto Superior Técnico, Technical University of Lisbon, Portugal \\ D. Camotim \\ Civil Engineering Department, ICIST, Instituto Superior Técnico, Technical University of Lisbon, Portugal \\ N. Silvestre \\ Civil Engineering Department, ICIST, Instituto Superior Técnico, Technical University of Lisbon, Portugal
}

\begin{abstract}
After providing a brief overview of a recently developed and validated elastoplastic post-buckling Generalised Beam Theory (GBT) formulation, the paper presents and discusses illustrative numerical results concerning three tubular members exhibiting bi-linear and non-linear material behaviours. The GBT results consist of equilibrium paths, modal participation diagrams, stress contours, displacement profiles and collapse mechanisms, most of which are compared with values obtained from ABAQUS shell finite element analyses. The GBT modal nature makes it possible to (i) acquire in-depth knowledge about the member behavioural mechanics at any given equilibrium state (elastic or elastic-plastic), as well as (ii) evidence the GBT computational efficiency (d.o.f. reduction of over 75\%), partly due to the exclusion from the analyses of all deformation modes playing no role in a given member response.
\end{abstract}

\section{INTRODUCTION}

Most thin-walled metal members exhibit slenderness values leading to structural behaviours and ultimate strengths governed by interaction between plasticity and instability, which renders their rigorous analysis a complex task. This stimulates the development of more efficient design rules and methods of structural analysis. In this context, even if conventional structural design concerns mostly serviceability/ultimate limit states, a performance-based design also requires accurate member post-collapse response predictions (Hiriyur \& Schafer 2005). As experimental studies are invariably scarce, alternative approaches must be sought. The most universal is performing non-linear shell finite element analyses (SFEA), involving (i) large degree of freedom (d.o.f.) numbers, (ii) time-consuming/error-prone data processing and interpretation, and (iii) nodal stress/displacement outputs, rather than modal ones (e.g., stress resultants).

Generalised Beam Theory (GBT) is an alternative to the above structural analysis methods. In spite of its limited current field of application (prismatic, straight and non-perforated thin-walled bars) and dissemination, GBT is recognised as an elegant and efficient approach to analyse thin-walled members and structural systems. These traits arise mostly from its modal nature: displacement field expressed as a linear combination of cross-section deformation modes with amplitudes varying continuously along the member length. This feature makes it possible to (i) acquire in-depth insight on the member behaviour and (ii) judiciously exclude from future similar analyses those modes found to play no role in the particular behaviour under scrutiny, thus further reducing the d.o.f. number. GBT has attracted the interest of several researchers, leading to new formulations and applications concerning various types of analysis, support and loading conditions, and materials (Camotim et al. 2010). With a few exceptions, the material models considered were linear elastic. The first physically non-linear GBT formulations are due to Gonçalves \& Camotim (2004, 2011, 2012): beam finite elements (BFE) based on $J_{2^{-}}$ flow plasticity theory aimed to perform member bifurcation, first and second-order inelastic analyses. The authors (Abambres et al. 2012, 2013a-c) developed and validated alternative $1^{\text {st }}$ and $2^{\text {nd }}$ order elastoplastic GBT formulations, also based on $J_{2}$-flow theory but differing from the previous ones in the (i) deformation mode determination and (ii) consideration of warping rotations (novel d.o.f.) - in these works, GBT was first applied to strain-hardening materials, namely stainless steel. When compared with carbon steel, stainless steel (i) has no welldefined yield point and (ii) exhibits a high non-linearity beyond the proportional limit.

The aim of this paper is (i) to present a brief overview of the materially and geometrically non-linear GBT formulation, and (ii) to illustrate its application and potential by studying the post-buckling behaviour and strength of tubular members made of perfectly-plastic, bi-linear and non-linear materials. The analyses include local and/or global initial geometrical imperfections, but neglect membrane residual stresses and corner strength effects. For validation, most GBT results (equilibrium paths, displacement profiles, stress contours, collapse mechanisms) are compared with ABAQUS SFEA values. The GBT modal nature is highlighted through the presentation of modal participation diagrams. 


\section{GBT NON-LINEAR ELASTIC-PLASTIC FORMULATION - OVERVIEW}

Only an overview of the main concepts and procedures involved in the development and implementation of the proposed GBT formulation is reported. After focusing on aspects related to kinematics and cross-section analysis, the member non-linear equilibrium equations are established and their BFE incremental solution addressed.

\subsection{Kinematics and Equilibrium Equations}

Consider the local coordinate system $(x, s, z)$ shown in Figure 1(a) at each wall mid-surface of a thin-walled bar, where $x, s$ and $z$ are the longitudinal $(0 \leq x \leq L-$ member length), transverse ( $0 \leq s \leq b-$ wall width) and throughthickness $(-t / 2 \leq z \leq t / 2-$ wall thickness) coordinates - the associated displacements are $u$ (warping), $v$ (transverse) and $w$ (flexural). The GBT analysis consists of two main steps: (i) cross-section analysis and (ii) member analysis. The former leads to the deformation mode displacement profiles $u_{k}, v_{k}$ and $w_{k}$ (for mode $k$ ), along $s$, while the member analysis determines the modal amplitude functions $\zeta_{k}(x)$-displacement profile longitudinal variations. Then, the GBT mid-surface displacement field is given by a linear combination of products between modal displacement profiles and their amplitude functions,

$$
\begin{aligned}
& u(s, x)=u_{k}(s) \zeta_{k, x}(x) \quad v(s, x)=v_{k}(s) \zeta_{k}(x) \\
& w(s, x)=w_{k}(s) \zeta_{k}(x)
\end{aligned}
$$

where $k$ satisfies the summation convention. The crosssection analysis adopted considers 4 deformation mode families: conventional, cell shear flow, warping shear and transverse extension. The existing analyses, considering either $3(u, v, w)$ or $4\left(u, v, w, \theta_{x}\right.$ - rotation about the $x$ axis) d.o.f. per section node, require fine discretisations to capture highly non-linear axial displacement variations along the cross-section mid-line, due to spread of yielding and/or shear deformation. This justifies the convenience of enhancing the warping representation, done here through additional nodal d.o.f. ("warping rotations"): rotations about the $z$-axis $\theta_{z}$ - one per intermediate node and more than one per natural node (independent $\theta_{z}$ per converging wall direction). This enables the approximation of each warping profile $u_{k}$ by piecewise (in each wall segment) cubic polynomials, instead of piecewise linear functions (see warping shear modes in Figs. 4 and 6).

Note that the formulation keeps the GBT plane-stress assumption (null $\sigma_{x z}, \sigma_{s z}, \sigma_{z z}$ stresses and $\gamma_{x z}, \gamma_{s z}, \varepsilon_{z z}$ strains). Accordingly, the Principle of Virtual Work leads to the GBT equilibrium equations ( $m, n=\{x, s\})$

$$
\iint_{L} \int_{b} \sigma_{t} \delta \varepsilon_{m n} d z d s d x=\iint_{L}\left[\begin{array}{l}
\left(q_{x} u_{i}\right) \delta \zeta_{i, x}+ \\
\left(q_{s} v_{i}+q_{z} w_{i}\right) \delta \zeta_{i}
\end{array}\right] d s d x
$$

where (i) $\sigma_{x x}, \sigma_{s s}, \sigma_{x s}$ are axial, transverse and shear $2^{\text {nd }}$ Piola-Kirchhoff stresses, (ii) $q_{x}, q_{s}, q_{z}$ are local compo- nents of a distributed load on the member mid-surface (see Fig. 1(b)), and (iii) $\delta \varepsilon_{x x}, \delta \varepsilon_{s s}, \delta \gamma_{x s}$ are axial, transverse and shear Green-Saint-Venant non-linear virtual strains, expressed in terms of the modal amplitude functions $\zeta_{k}(x)$ and their derivatives, through Eqs. (1).The formulation also accounts for (i) residual stresses and geometrical imperfections, and (ii) arbitrary single-parameter $(\lambda)$ loadings, including concentrated forces and moments.

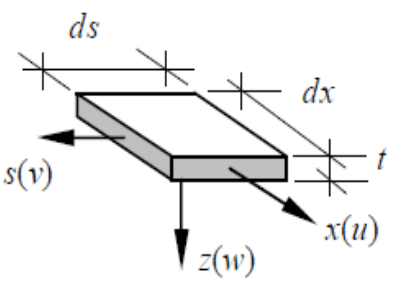

(a)

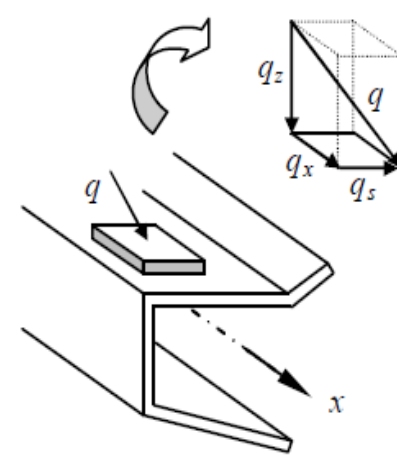

(b)
Figure 1. (a) Local coordinate system at each wall mid-surface and (b) general external distributed load $q(x, s)$.

\subsection{Non-Linear Beam Finite Element}

The cylindrical arc-length method is adopted, involving (i) incremental equilibrium equations based on the tangent stiffness matrix $K_{\text {tan }}$, (ii) internal force vectors $f^{\text {int }}$ and (iii) a path-independent iterative strategy: strain increments evaluated w.r.t. the last converged equilibrium state.

The results shown in section 3 were obtained using Hermite cubic polynomials to approximate the GBT modal amplitude functions in each BFE, i.e.,

$$
\zeta_{k, x}^{v, w=0}(x)=\Psi_{H}(x) d_{k} \quad \zeta_{k}^{v, w \neq 0}(x)=\Psi_{H}(x) d_{k}
$$

where (i) the $1 \times 4 \Psi_{H}$ vector stores the Hermite polynomials, (ii) $d_{k}$ is the $4 \times 1$ displacement vector related to the mode $k$ approximation, (iii) the first expression concerns only axial extension and warping shear modes $(v=w=0)$, and (iv) the second expression applies to all other modes $(v \neq 0$ and/or $w \neq 0)$. After inserting the above approximations in Eq. (2) (including virtual strains), the internal and external force vectors interrelation for a generic "equilibrium configuration" $(m n=x x, s s, x s)$

$$
\sum_{m n} f_{i(m n)}^{\mathrm{int}}=\lambda \bar{f}_{i}, \quad f_{i(m n)}^{\mathrm{int}}=\int_{L_{e}} \int_{b} \int_{t} \sigma_{m n} F_{i(m n)}^{\mathrm{int}} d V_{e}
$$

is obtained, where (i) the $i^{\text {th }}$ components are $4 \times 1$ subvectors related to deformation mode $i$ and (ii) $\bar{f}$ is the external force vector for a unit load parameter $\lambda$. Then, the incremental equilibrium equation for a deformed configuration $j$ can be established as

$$
\left.K_{\tan }\right|_{j} \Delta d=\Delta \lambda \bar{f}
$$

where (i) $d$ is the displacement vector and (ii) the tangent stiffness matrix $(i, p)^{\text {th }}$ component, $K_{i p \text {, tan }}$, is a $4 \times 4$ submatrix concerning deformation modes $i$ and $p$, reading 


$$
\begin{aligned}
& K_{i p, \tan }=\frac{\partial f_{i}^{\mathrm{int}}}{\partial d_{p}}, \quad \frac{\partial f_{i}^{\mathrm{int}}}{\partial d_{p l}}=\frac{\partial f_{i(x x)}^{\mathrm{int}}}{\partial d_{p l}}+\frac{\partial f_{i(s s)}^{\mathrm{int}}}{\partial d_{p l}}+\frac{\partial f_{i(x s)}^{\mathrm{int}}}{\partial d_{p l}} \\
& \frac{\partial f_{i(m n)}^{\mathrm{int}}}{\partial d_{p l}}=\int_{L_{e}} \int_{b} \int_{t}\left\{\frac{\partial \sigma_{m n}}{\partial d_{p l}} F_{i(m n)}^{\mathrm{int}}+\sigma_{m n} \frac{\partial F_{i(m n)}^{\mathrm{int}}}{\partial d_{p l}}\right\} d V_{e}
\end{aligned}
$$

It corresponds to the Jacobian of the internal force vector $i^{\text {th }}$ component, w.r.t. the mode $p$ displacement vector - its components are $d_{p l}(l=1, \ldots, 4)$. For any inelastic material, the stress gradients at every point read

$$
\frac{\partial \sigma_{m n}}{\partial d_{p l}}=\frac{\partial \sigma_{m n}}{\partial \varepsilon_{x x}} \frac{\partial \varepsilon_{x x}}{\partial d_{p l}}+\frac{\partial \sigma_{m n}}{\partial \varepsilon_{s s}} \frac{\partial \varepsilon_{s s}}{\partial d_{p l}}+\frac{\partial \sigma_{m n}}{\partial \gamma_{x s}} \frac{\partial \gamma_{x s}}{\partial d_{p l}}
$$

$\left(\partial \varepsilon / \partial d_{p l}\right.$ obtained from writing the strain-displacement relations according to the FE approximations in Eq. (3)).

The stresses and their gradients $(\partial \sigma / \partial \varepsilon)$ appearing in previous relations stem from the $J_{2}$-flow theory with associated flow rule, by resorting to implicit and/or explicit numerical integration schemes wherever plastic flow takes place. Then, at every Gauss point and after each arclength iteration, one must (i) compute the elastic stress variation, from the imposed strain variation $(\Delta \varepsilon)$ w.r.t. the last equilibrium state $i$ (stresses $\sigma_{i}$ and hardening parameter $\kappa_{i}$ ), and then (ii) check whether the final stresses $\left(\sigma_{e}=\sigma_{i}+D^{e} \Delta \varepsilon\right)$ fall outside the yield surface $f\left(\sigma, \kappa_{i}\right)=0$. If yes, numerical integration of the incremental constitutive equations is performed to update the stresses and hardening parameter $\kappa$ - the Mean-Normal and EulerBackward methods were used to obtain the results presented in 3.2 and 3.3-3.4, respectively. Once those variables are updated, gradients $\partial \sigma / \partial \varepsilon$ are directly computed from the consistent elastoplastic constitutive matrix.

\section{ILLUSTRATIVE EXAMPLES}

The application of the GBT formulation for inelastic postbuckling analysis, implemented in MATLAB (MathWorks 2010), is now illustrated. The results discussed next concern 3 numerical examples involving (i) SHS and hollow flange (HF) I-section columns and (ii) a RHS beamcolumn. Material models with a linear elastic regime and involving different types of strain-hardening were considered, namely the (i) perfectly-plastic (PP), (ii) bi-linear (BL-linear hardening), and (iii) non-linear (NL) models. Concerning the latter, the $\sigma-\varepsilon$ relation due to Quach et al. (2008) is adopted - it is (i) suitable for stainless steel alloys and (ii) defined by the 3 basic Ramberg-Osgood parameters $\left(E, \sigma_{0.2}, n\right)$ (implementation details reported in Abambres et al. 2013c). For validation purposes, GBT equilibrium paths, stress contours and deformed configurations (referred to the bar mid-surface) are compared with those yielded from ABAQUS (DS Simulia 2008) SFEA.

Table 1 shows the material properties adopted in each analysis: (i) $E, E_{\text {sh }}$, are the Young's modulus and strainhardening slope (BL model), (ii) $\sigma_{0}^{\mathrm{y}}, \sigma_{0.2}$, are the initial yield and $0.2 \%$ proof stresses, and (iii) $n$ is the hardening power (NL model $-0.01 \%$ proof stress taken as the initial yield stress) $-c, t$ denote compressive and tensile. All these (isotropic) models are employed to simulate the compressive behaviour and a $v=0.3$ Poisson's ratio is assumed. Table 2 shows the numbers of (i) GBT BFEs, (ii) axial and transverse $(x, s)$ ABAQUS SFEs, and (iii) d.o.f. involved in each model. As for the numbers of Gauss integration points per BFE wall segment direction $s, z, x$ (Fig. 1(a)) in each GBT analysis, they are (i) $(3,3,3)$ for the SHS, (ii) $(4,5,3)$ for the HF I-section and (iii) $(2,5,4)$ for the RHS - the SFEA involved fairly uniform S4 FE meshes, adopting the same through-thickness integration points as the GBT analyses.

Table 1: Illustrative examples: material properties

\begin{tabular}{cccccc}
\hline Example & $E(\mathrm{GPa})$ & $E_{s h}$ & $\sigma_{0}{ }^{\mathrm{y}}(\mathrm{MPa})$ & $\sigma_{0.2}(\mathrm{MPa})$ & $n$ \\
\hline SHS & $\begin{array}{c}E^{c}=198.2 \\
E^{t}=198.8\end{array}$ & - & 390.3 & $\begin{array}{c}\sigma_{0.2}{ }^{c}=560 \\
\sigma_{0.2}{ }^{t}=586\end{array}$ & $\begin{array}{c}n^{c}=8.3 \\
n^{t}=9.0\end{array}$ \\
\hline $\begin{array}{c}\text { HF I-sec } \\
(P P \& B L)\end{array}$ & 205 & $E / 50$ & 290 & - & - \\
\hline $\begin{array}{c}\text { HF I-sec } \\
(N L)\end{array}$ & $\begin{array}{c}E^{c}=205 \\
E^{t}=208\end{array}$ & - & 289.5 & $\begin{array}{c}\sigma_{0.2}{ }^{c}=515 \\
\sigma_{0.2}=560\end{array}$ & $\begin{array}{c}n^{c}=5.2 \\
n^{t}=6.9\end{array}$ \\
\hline RHS & 200 & 0 & 450 & - & - \\
\hline
\end{tabular}

Table 2: Illustrative examples: FE discretisation and d.o.f. numbers.

\begin{tabular}{c|ccc|ccc}
\hline \multirow{2}{*}{ Example } & GBT & \multicolumn{2}{|c|}{ ABAQUS } & \multicolumn{3}{c}{ Number of d.o.f. } \\
\cline { 2 - 7 } & BFEs & $\begin{array}{c}\text { SFEs } \\
(x)\end{array}$ & $\begin{array}{c}\text { SFEs } \\
(s)\end{array}$ & GBT & ABQ & $\begin{array}{c}\text { GBT/ABQ } \\
(\%)\end{array}$ \\
\hline SHS & 16 & 50 & 48 & 865 & 14401 & 6 \\
\hline HF I-sec & $66(*)$ & 80 & 62 & 7391 & 29651 & 24.9 \\
\hline RHS & 50 & 160 & 36 & 6462 & 34452 & 18.8 \\
\hline
\end{tabular}

*Only non-uniform (symmetric) mesh: 18 BFEs $(x<0.35 L)+15$ BFEs $(0.35 L \leq x \leq 0.5 L)$

After providing information about the selected GBT modes, in 3.1, the results of each analysis are addressed in 3.2-3.4. The first one concerns a fixed-ended SHS stub column (Fig. 3(a) $-L=400 \mathrm{~mm}$ ), made of EN $1.4162 \mathrm{du}-$ plex stainless steel and acted by a (uniformly distributed) compressive end force $\mathrm{F}=102.2 \times 10^{4} \lambda \mathrm{N}$ (experimental results reported by Theofanous \& Gardner 2009). The initial geometrical imperfection, akin to the $1^{\text {st }}$ symmetric local buckling mode, has a $t / 100$ amplitude (maximum in-plane displacement). The second problem deals with a pin-ended HF I-section column (Fig. 3(b) $L=1200 \mathrm{~mm}$ ) acted by a compressive end force $\mathrm{F}=100 \times 10^{3} \lambda \mathrm{N}$ - besides the end-shortening, the pinned supports allow for global flexural rotations. In order to assess the impact of strain-hardening on the column behaviour and strength, analyses were carried out for PP, BL and NL material models with the same initial yield stress - Figure 2 shows their $\sigma-\varepsilon$ curves. The initial imperfections combine the $1^{\text {st }}$ global (flexural $-L / 1000$ amplitude) and 13 half-wave local (1.18 $\mathrm{mm}$ amplitude) buckling modes. Lastly, a fixed-pinned (no in-plane motions) RHS beam-column (Fig. 3(c) $-L=4000 \mathrm{~mm}$ ) made of a PP material is analysed, subjected to (i) a uniformly distributed vertical load acting along the full top flange $\left(p=0.01 \lambda \mathrm{N} / \mathrm{mm}^{2}\right)$ and (ii) an axial compression $\mathrm{F}=100 \times 10^{3} \lambda \mathrm{N}$. The imperfection is akin to the $2^{\text {nd }}$ local buckling mode and has amplitude equal to $t / 100$. 


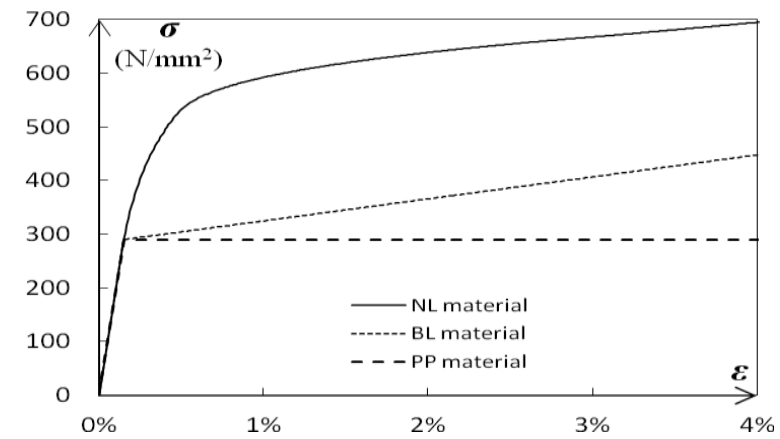

Figure 2. HF I-section column material model (stress-strain curves).

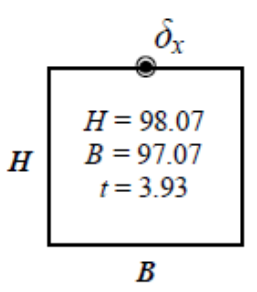

(a) fixed-ended

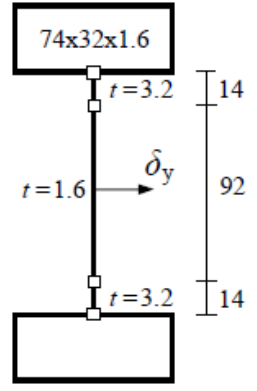

(b) pin-ended

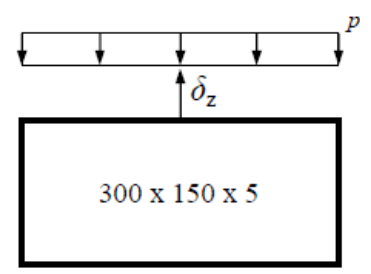

(c) fixed-pinned
Figure 3. (a) SHS, (b) HF I-section, (c) RHS mid-line dimensions.

The results shown in 3.2-3.4 correspond to the points indicated on the equilibrium path, denoted as BP (before peak), P (peak) and AP (after peak). It is also worth noting that the GBT modal participation diagrams are based on the cross-section having the maximum overall or inplane displacement (initial imperfection not included) each deformation mode contribution is the ratio between its maximum displacement and the sum of all such modal values, not necessarily occurring at the same point.

\subsection{GBT deformation modes}

The GBT deformation modes (G-global, D-distortional, L-local, WS-warping shear, TE-transverse extension and CS-non-global cell shear flow) included in each analysis are the following: (i) 28 modes out of 204 ( 1 G, 6 L, 11 WS, $10 \mathrm{TE}$ ), all bi-symmetric (SHS), (ii) 56 modes out of 132 (2 G, 1 D, 15 L, 20 WS, 17 TE, 1 CS), mostly symmetric w.r.t. the horizontal centroidal axis (HF I-section), and (iii) 65 modes out of 184 ( 2 G, 20 L, 18 TE, 25 WS), symmetric w.r.t. the vertical centroidal axis (RHS) - all these

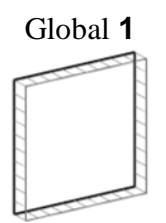

Local 4

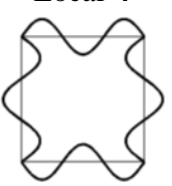

Transverse Ext. 19

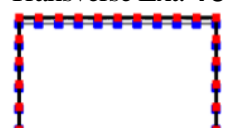

Local 2

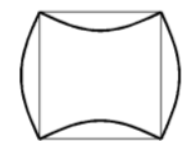

Warping Shear 8

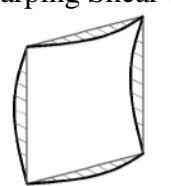

Transverse Ext. 20

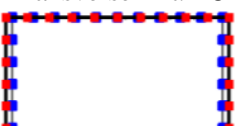

Local 3

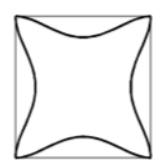

Warping Shear 9

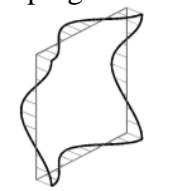

Transverse Ext. 22

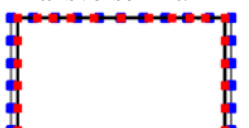

Figure 4. SHS most relevant GBT deformation modes.

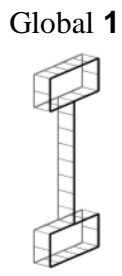

Local 7 Local 8
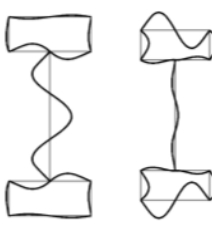

Local 9
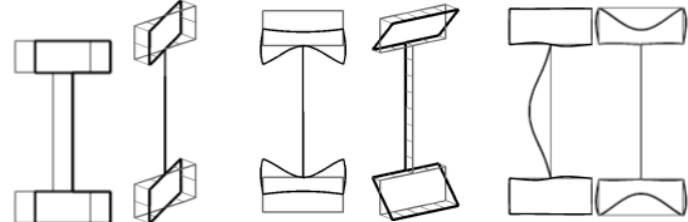

Warping Shear 22, 23, 24, 26
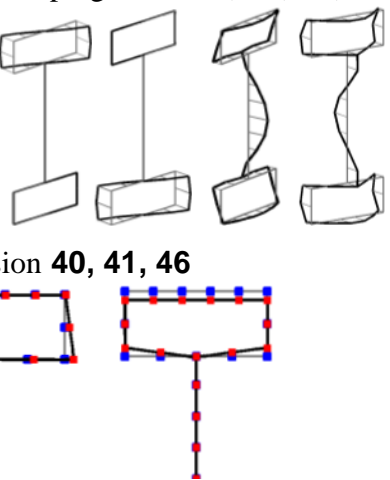

Transverse Extension 40, 41, 46
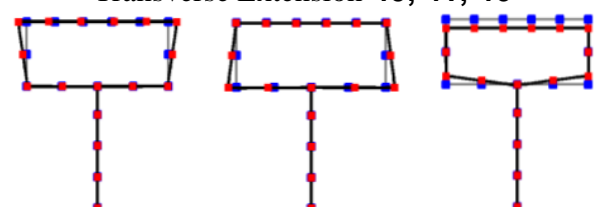

Figure 5. HF I-section most relevant GBT deformation modes.

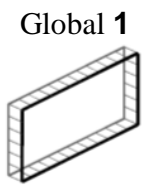

Global 2
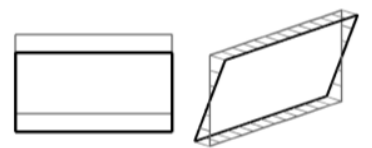

Local 3

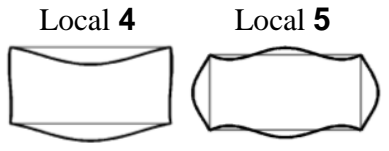

Warping Shear 23, 25
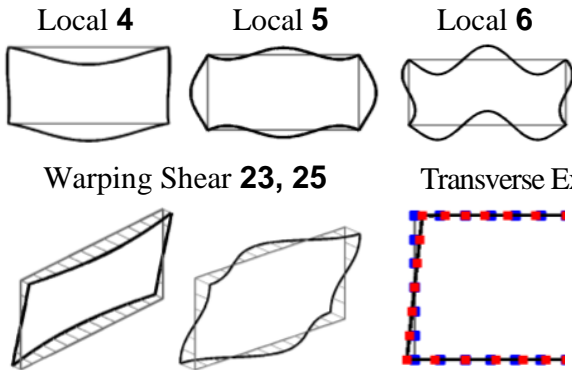

Transverse Extension. 51, 52
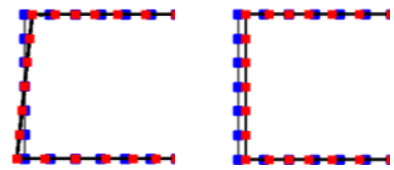

Figure 6. RHS most relevant GBT deformation modes.

modes are renumbered sequentially, since they are just a fraction of the cross-section analysis full set. The in/outof-plane shapes of the most relevant modes in each analysis are shown in Figures 4-6 - the discretisations adopted are shown in the symmetric TE mode half-section shapes - warping rotation d.o.f. $\theta_{z}$ (addressed in subsection 2.1) were used in the SHS and RHS member analyses.

\subsection{SHS column}

Figure 7(a) shows the GBT and ABAQUS $\lambda(\Delta)$ equilibrium paths ( $\Delta$ is the column end shortening) - the $\mathrm{BP}, \mathrm{P}$ and $\mathrm{AP} \lambda$ values are: $\lambda_{\mathrm{GBT}}=0.701+\lambda_{\mathrm{ABQ}}=0.701(\mathrm{BP})$, $\lambda_{\mathrm{GBT}}=0.893+\lambda_{\mathrm{ABQ}}=0.893(\mathrm{P}), \lambda_{\mathrm{GBT}}=0.590+\lambda_{\mathrm{ABQ}}=0.577$ (AP). Fig. 7(b) shows the GBT modal participations concerning the evolution, with $\Delta$, of the mid-span crosssection deformed shape. The following remarks are due:

(i) The agreement between the GBT and ABAQUS paths is very good (differences below $4.9 \%$ ).

(ii) The GBT participation diagram indicates two markedly different behaviours: (ii 1 ) one prior to the peak, involving mostly mode 1 (58.6-84.7\%), and ( $\mathrm{ii}_{2}$ ) another after the peak: growth of local mode 2 (27.164.5\%), "sweeping aside" mode 1. 
(a)

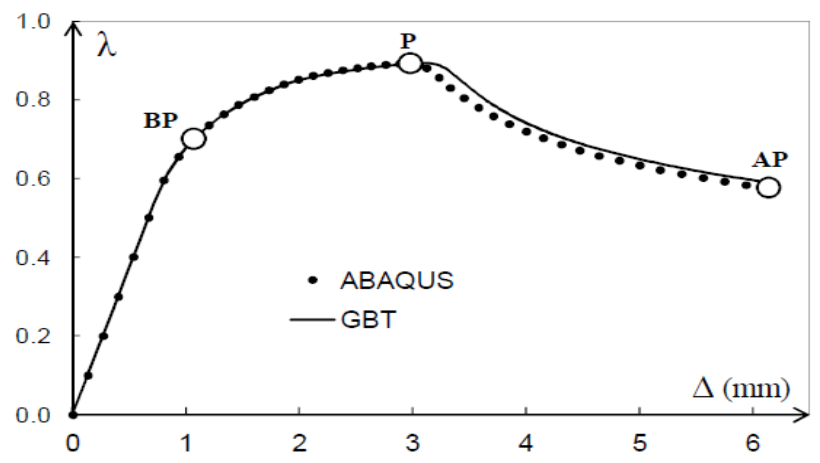

(b)

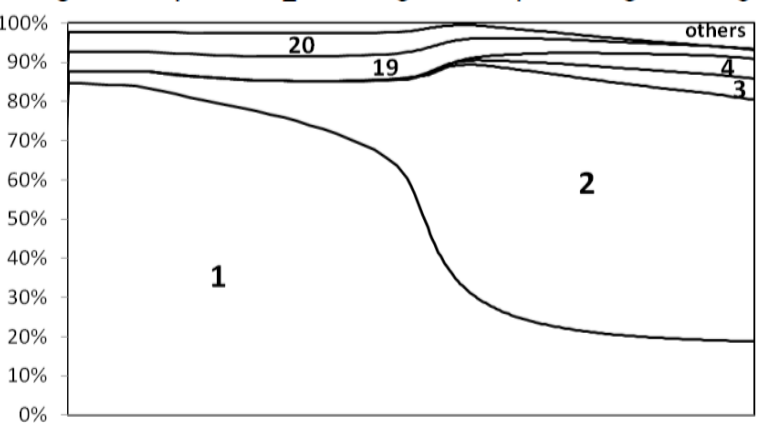

Figure 7 (a) GBT and ABAQUS equilibrium paths and (b) GBT modal participation diagram.

(iii) Concerning the TE modes 19 and 20, whose presence is intrinsically linked to modes 1 (Poisson effects) and 2, it should be noted that, in spite of their small contributions ( $\approx 6 \%$ each), they play a key role. Indeed, their removal from the analysis would considerably stiffen the column behaviour since local buckling would be "artificially restrained". The postpeak participation decrease of these TE modes stems from elastic unloading and local deformation "localisation" very close to mid-height (see Fig. 8).
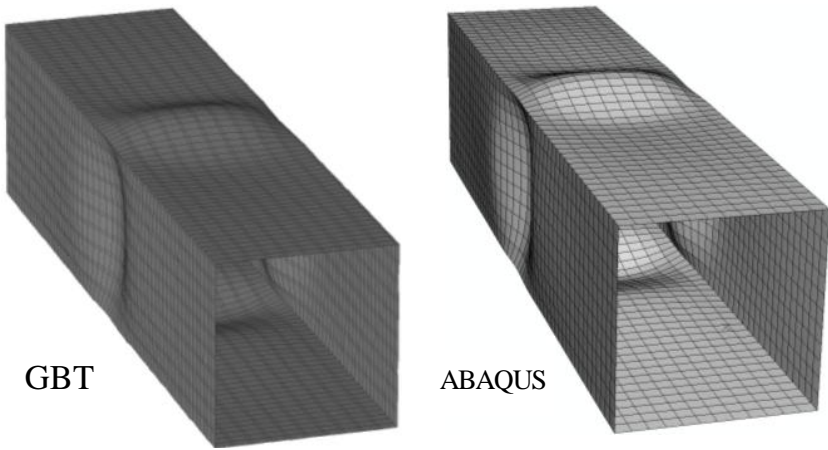

Figure 8.Column collapse mechanism (AP equilibrium state).

Figure 9 depicts the BP, $\mathrm{P}$ and AP axial displacement profiles $\delta_{x}(x)$ of the top wall mid-point (see Fig. 3(a)). First of all, it is readily observed that the ABAQUS and GBT results virtually coincide at the BP and AP states, and exhibit small differences at the $\mathrm{P}$ state. Moreover, note the linearity of the BP profile, while its $\mathrm{P}$ counterpart exhibits a mild non-linearity close to the column ends. At the AP state, on the other hand, $\delta_{x}$ exhibits three "almost linear" segments, the two outer ones with similar (small) slopes and a much steeper inner one - this axial stiffness drop occurring in the column central region stems from the plastic deformations associated with the increasing participations of the local modes 2, 3 and $\mathbf{4}$ (see Fig. 7(b)).

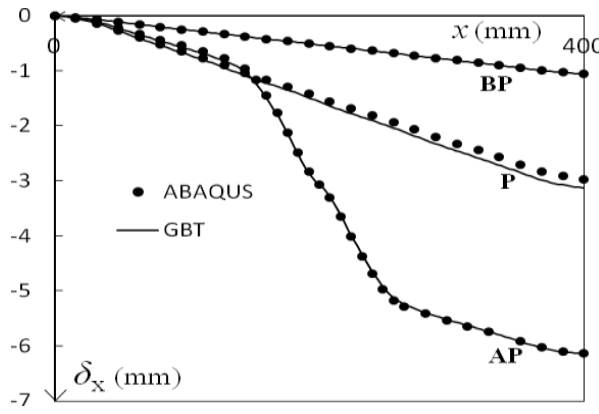

Figure 9. Axial displacement profiles of the top wall mid-point at the $\mathrm{BP}, \mathrm{P}$ and $\mathrm{AP}$ equilibrium states.

\subsection{HF I-section column}

Figure 10(a) shows the GBT and ABAQUS $\lambda\left(\delta_{\mathrm{y}}\right)$ equilibrium paths concerning the $\mathrm{PP}, \mathrm{BL}$ and NL material models $\left(\delta_{\mathrm{y}}\right.$ is the mid-span cross-section mid-height lateral displacement - see Fig. 3(b)). For the NL paths, the BP, $\mathrm{P}$ and $\mathrm{AP}$ applied load values are $\lambda_{\mathrm{GBT}}=2.79+\lambda_{\mathrm{ABQ}}=2.76$ (BP), $\lambda_{\mathrm{GBT}}=3.08+\lambda_{\mathrm{ABQ}}=3.05(\mathrm{P}), \lambda_{\mathrm{GBT}}=1.99+\lambda_{\mathrm{ABQ}}=1.94$ (AP). As for Figure 10(b), it provides the evolution, with $\delta_{\mathrm{y}}$, of the mid-span cross-section deformed configuration (via the GBT modal participation diagram). Note that:

(i) Except for the PP material at advanced post-collapse stages, the GBT and ABAQUS curves agree very well - e.g., the NL paths do not differ by more than $4 \%$.

(ii) The PP and BL column responses prior to the later collapse stages virtually coincide (in spite of the inelastic collapse). However, the behaviour is much stiffer for the NL column $\left(\lambda_{\mathrm{u}, \mathrm{NL}}=3.05, \lambda_{\mathrm{u}, \mathrm{BL}}=2.28\right)$, due to the large strain-hardening (partly responsible for the high non-linearity prior to reaching the peak load).

(iii) Figure 10(b) shows that the column behaviour exhibits relevant contributions from ( $\mathrm{iii}_{1}$ ) global modes 1 and 2 (21.2-69.7\%), and (iii 2 ) local modes 5 (8.9$36.1 \%), 6$ and 8+9. Although the web-driven mode 5 dominates the local deformation in the path ascending

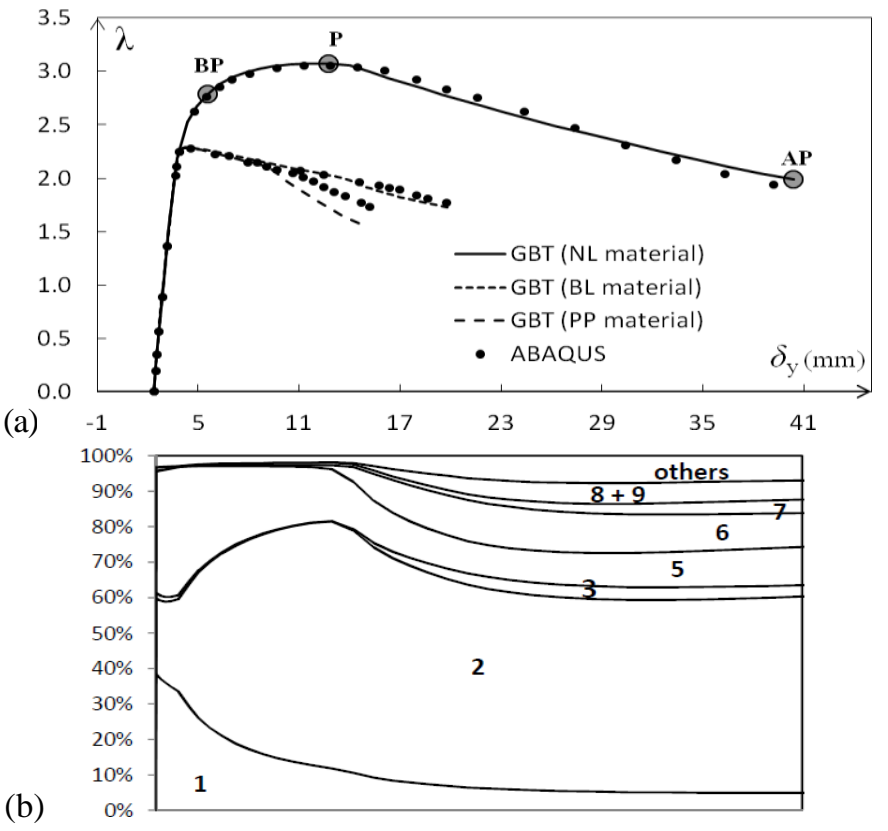

Figure 10 (a) GBT and ABAQUS equilibrium paths and (b) GBT modal participation diagram (NL column). 

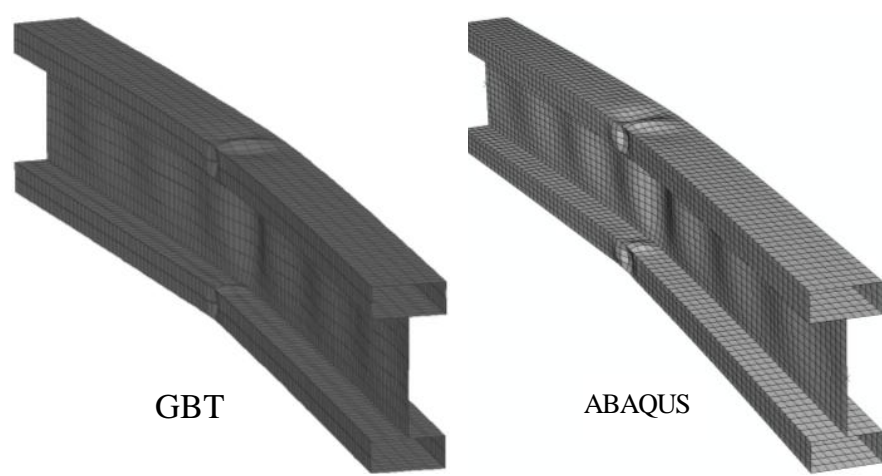

Figure 11.Column failure mechanism (AP equilibrium state).

branch (recall it participates in the initial imperfection), important contributions from the flangedriven modes $\mathbf{6}, \mathbf{8}$ and $\mathbf{9}$ occur in the post-collapse phase - this can be attested by the failure mechanism (AP state) depicted in Fig. 11 (note the yield-line forming at the most compressed flange regions).

Figure 12 concerns the NL material response at the $\mathrm{BP}, \mathrm{P}$ and AP states and shows the web mid-height lateral displacement profiles $\delta_{\mathrm{y}}(x)$ (Fig. 3(b)). The agreement between GBT and SFEA results is fairly good visible discrepancies stem mostly from differences in the initial imperfection prediction. In order to assess the material model influence, Figure 13 compares the GBT $\delta_{\mathrm{y}}(x)$ profiles for $\lambda_{\mathrm{GBT}}=1.99$ (AP state) - it is observed that, in spite of the expected quantitative differences, they all follow the same trend (same number of half-waves).

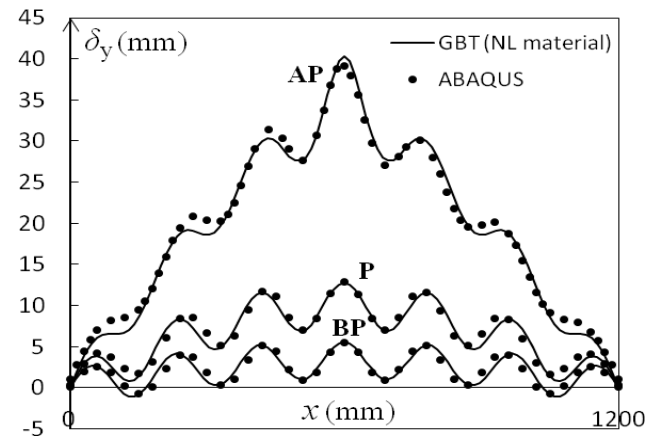

Figure 12. Cross-section mid-height lateral displacement profiles of the NL column at the BP, P and AP equilibrium states.

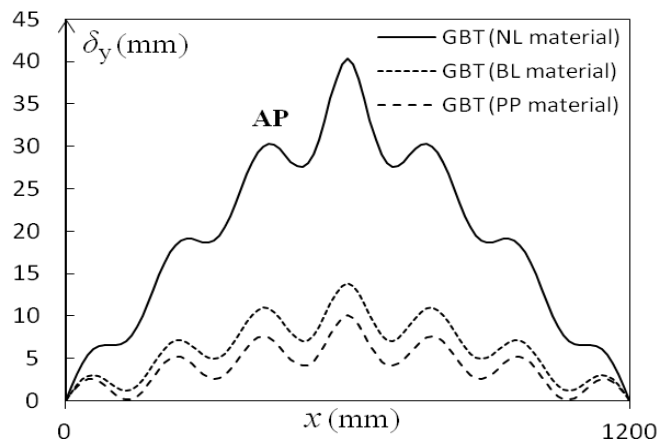

Figure 13. Cross-section mid-height lateral displacement profiles at $\lambda_{\mathrm{GBT}}=1.99$ for the NL, BL and PP columns.

\subsection{RHS beam-column}

Figure 14(a) shows the GBT and ABAQUS $\lambda\left(\left|\delta_{z}\right|\right)$ equilibrium paths $\left(\delta_{\mathrm{Z}}\right.$ is the mid-span top flange mid-point verti- cal displacement - Fig. 3(c)). The BP, $\mathrm{P}$ and AP $\lambda$ values are $\lambda_{\mathrm{GBT}}=7.51+\lambda_{\mathrm{ABQ}}=7.50(\mathrm{BP}), \lambda_{\mathrm{GBT}}=8.38+\lambda_{\mathrm{ABQ}}=8.43$ (P), $\lambda_{\mathrm{GBT}}=5.09+\lambda_{\mathrm{ABQ}}=4.81$ (AP). Figure 14(b) shows the modal participation diagram providing the evolution of the most deformed cross-section shape. It is possible to draw the following conclusions:

(i) The GBT and ABAQUS curves show a very good correlation in most of the deformation range. Even if the differences increase as loading progresses, they never exceed $6 \%$ (5\% excluding the last 2 ABAQUS points).

(ii) Figure 14(b) clearly shows that the early loading stages are governed by axial extension and minoraxis bending (modes 1 and $2-65.7-72.7 \%$ ). The local modes 3 (7.0-12.5\%) and 4 (4.6-10.5\%), which stem from the loaded top flange transverse bending and oppose each other in the bottom flange (Fig. 6), also have visible contributions. Lastly, mode 23 (one of the "others") captures the shear deformation associated with minor-axis bending.

(iii) In the post-peak stage there is a clear global deformation decrease (modes $\mathbf{1 + 2}$ ), mostly compensated by the gradual growth, for $\left|\delta_{z}\right| \geq 20 \mathrm{~mm}$, of local modes 5 (web-driven - 0.3-7.6\%) and 6+8 (1.0$4.7 \%$ ). The presence of local deformation in the member yield-line mechanism can be confirmed in Figure 15.

(a)

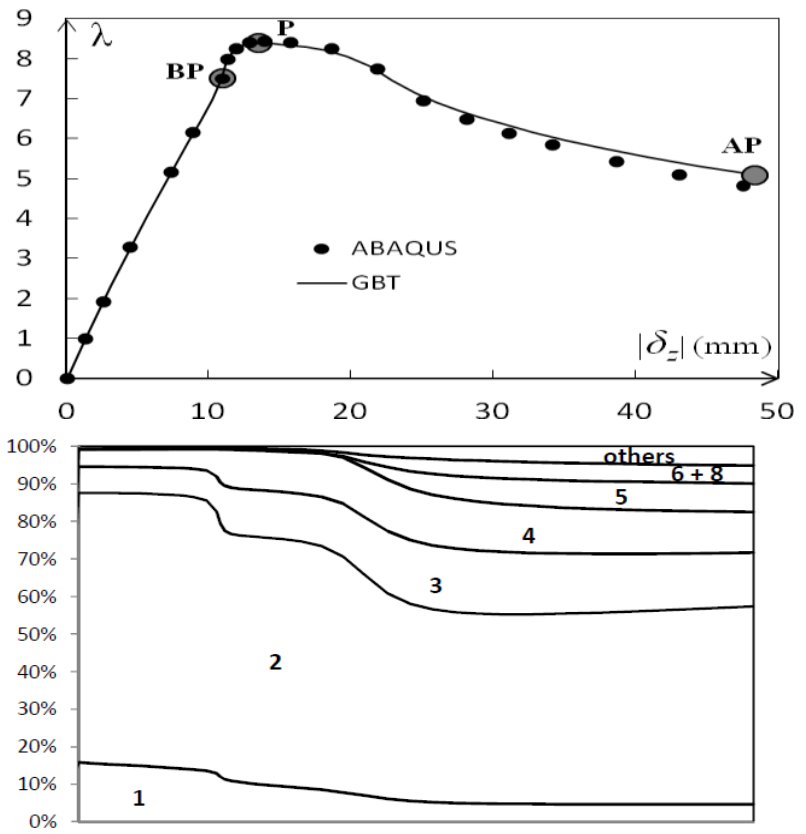

Figure 14 (a) GBT and ABAQUS equilibrium paths and (b) GBT modal participation diagram.

Figure 16 compares the top flange mid-point vertical displacement profiles at the $\mathrm{BP}, \mathrm{P}$ and $\mathrm{AP}$ states. Besides the excellent agreement between the GBT and ABAQUS outputs, note that the half-wave patterns change significantly in-between states P and AP, due to the plastic flow and elastic unloading associated with the failure mechanism. Finally, the half-column Mises stress contours depicted in Figure 17, concerning state $\mathrm{P}$ and evidencing excellent agreement, make it possible to visualise the most yielded (and compressed) flange regions at collapse: the vicinities of the (i) fixed end section bottom flange $(x \leq L / 2)$ and (ii) most deformed cross-section top flange $(x \geq L / 2)$. 

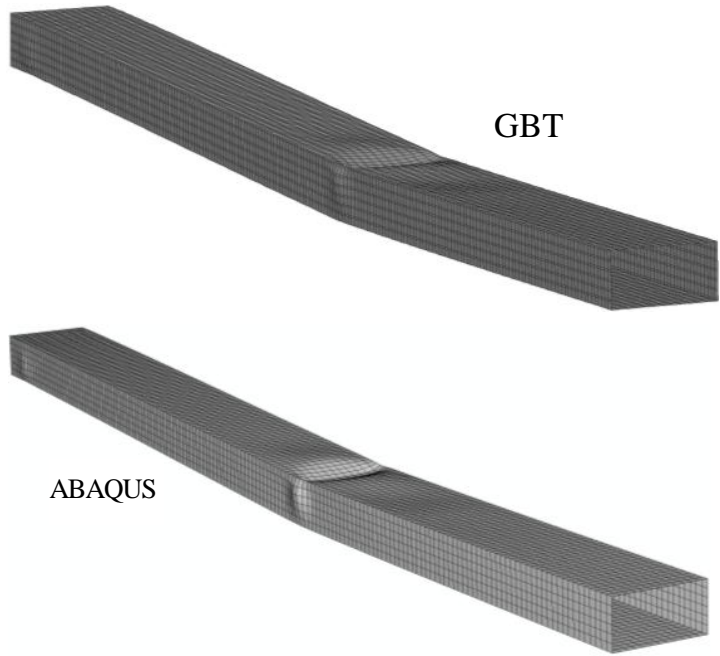

Figure 15. Member collapse mechanism (AP equilibrium state).

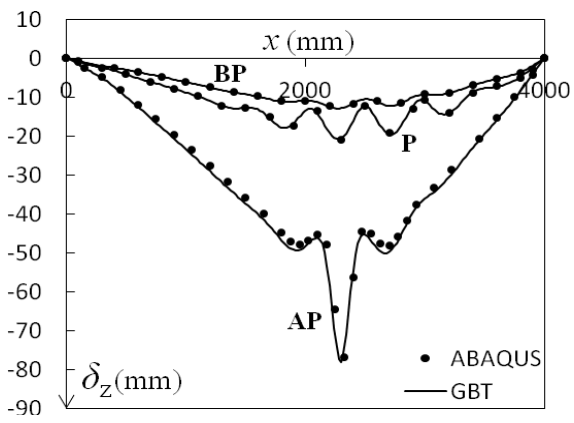

Figure 16. Vertical displacement profiles at the top flange middle point $-\mathrm{BP}, \mathrm{P}$ and AP states.

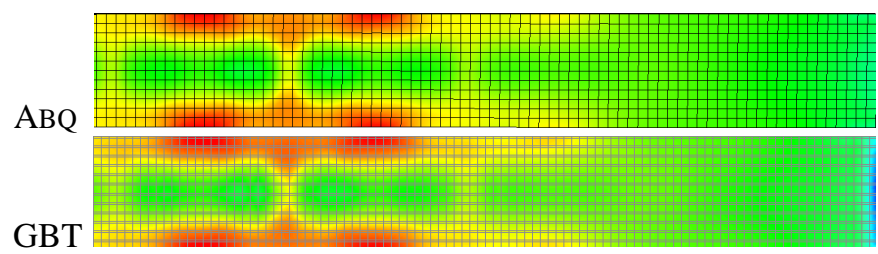

top flange $(x \geq L / 2-$ right half-column)

$\mathrm{ABQ}$

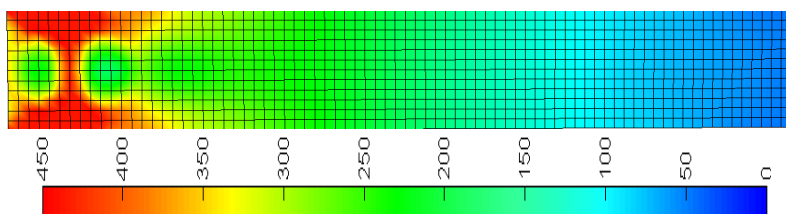

GBT

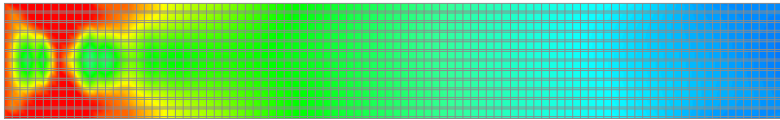

bottom flange ( $x \leq L / 2-$ left half-column)

Figure 17. Mises stress $\left(\mathrm{N} / \mathrm{mm}^{2}\right)$ contours at the P equilibrium state.

\section{CONCLUDING REMARKS}

This paper (i) provided an overview of a recently developed and validated GBT formulation to assess the inelastic post-buckling behaviour of thin-walled members, and (ii) illustrated its application and potential by presenting and discussing the analysis of several tubular members, namely a stainless steel SHS stub column, a perfectly plastic RHS beam-column and three hollow-flange Isection columns made of perfectly plastic, bi-linear and non-linear materials with identical yield stresses - the non-linear material exhibited a large strain-hardening and led to a significant strength increase. For validation purposes, most GBT results (equilibrium paths, collapse mechanisms, displacements profiles and stress contours) were compared with values yielded by ABAQUS shell finite element analyses. It was shown that the GBT analyses provide similarly accurate results, while requiring no more than $25 \%$ (16.6\% on average) of the d.o.f. numbers involved in the corresponding SFEA. Moreover, it was also shown, through modal participation diagrams, that the GBT analyses provide in-depth insight on the member mechanics associated with both the elastic and elastic-plastic regimes (including the collapse mechanisms).

\section{ACKNOWLEDGEMENTS}

The authors gratefully acknowledge the financial support of Fundação para a Ciência e Tecnologia (FCT - Portugal), through project PTDC/ECM/108146/2008. The first author also acknowledges FCT for granting his doctoral scholarship SFRH/BD/43271/2008.

\section{REFERENCES}

Abambres M., Camotim D. \& Silvestre N. 2012. Physically non-linear GBT analysis of thin-walled members, submitted for publication.

Abambres M., Camotim D. \& Silvestre N. 2013a. GBT-based firstorder analysis of elastic-plastic thin-walled steel members exhibiting strain-hardening, IES Journal A: Civil and Structural Engineering (Singapore), in press.

Abambres M., Camotim D. \& Silvestre N. 2013b. GBT-based structural analysis of elastic-plastic thin-walled members, Proceedings of SSRC Annual Stability Conference (St. Louis, 16-20/4).

Abambres M., Camotim D. \& Silvestre N. 2013c. GBT-based elasticplastic post-buckling analysis of stainless steel thin-walled members, submitted for publication.

Camotim D., Basaglia C., Bebiano R., Gonçalves R. \& Silvestre N. 2010. Latest developments in the GBT analysis of thin-walled steel structures, Proceedings of International Colloquium on Stability and Ductility of Steel Structures (SDSS'Rio 2010, 8-10/9), E. Batista et al. (eds.), 33-58.

DS Simulia Inc. 2008. Abaqus Standard Manual (version 6.7-5).

Gonçalves R. \& Camotim D. 2004. GBT local and global buckling analysis of aluminium and stainless steel columns, Computers and Structures, 82(17-19), 1473-1484.

Gonçalves R. \& Camotim D. 2011. Generalised beam theory-based finite elements for elastoplastic thin-walled metal members, ThinWalled Structures, 49(10), 1237-1245.

Gonçalves R. \& Camotim D. 2012. Geometrically non-linear generalised beam theory for elastoplastic thin-walled metal members, ThinWalled Structures, 51(February), 121-129.

Hiriyur B. \& Schafer B.W. 2005. Yield-line analysis of cold-formed steel members, International Journal of Steel Structures, 5(1), 43-54.

MathWorks. 2010. MATLAB - The Language of Technical Computing (www.mathworks.com/products/matlab).

Quach W.M., Teng J.G., Chung K.F. 2008. "Three-stage full-range stress-strain model for stainless steels", Journal of Structural Engineering (ASCE), 134(9), 1518-1527.

Theofanous M. \& Gardner L. 2009. "Testing and numerical modelling of lean duplex stainless steel hollow section columns", Engineering Structures, 31(12), 3047-3058. 